\title{
MOSAiC
}

\section{MOSAiC goes O2A - Arctic Expedition Data Flow from Observations to Archives and Analysis}

A. Immerz, M. Ajjan, J. Bein, B. Bräuer, T. Dinter, A. Driemel, T. Duede, J. Eilers, P. Gerchow, F. O. Glöckner, M. Günster, A. Haas, N. Harms, S. Immoor, R. Koppe, H. Liegmahl-Pieper, A. Macario, S. Makedanz, J. Matthes, M. Petri, H. Pfeiffenberger, S. Pinkernell, D. Ransby, S. Rehmcke, A. Schaefer, C. Schäfer-Neth, J. Schlüter, S. Schumacher, R. Spettnagel, A. Steinbach, A. Thiele, F. Thiele-Wolff, M. Thoma, A. Walter, P. Weidinger, S. Frickenhaus

Alfred Wegener Institute Helmholtz Centre for Polar and Marine Research Bremerhaven 
- Largest Arctic research expedition ever drifting Observatory for the Study

- to describe the processes coupling the atmosphere, sea ice, and ocean

- large suite of in-situ and remote sensing observations of physical, ecological and biogeochemical parameters

- Integrates between disciplines and between observations \& models

- Will lead to breakthrough in Arctic climate science.

- More than 60 institutions, 17 nations

- A total of $\sim 600$ people will operate in the central Arctic

- estimated up to 600 to be registered data of Arctic Climate users

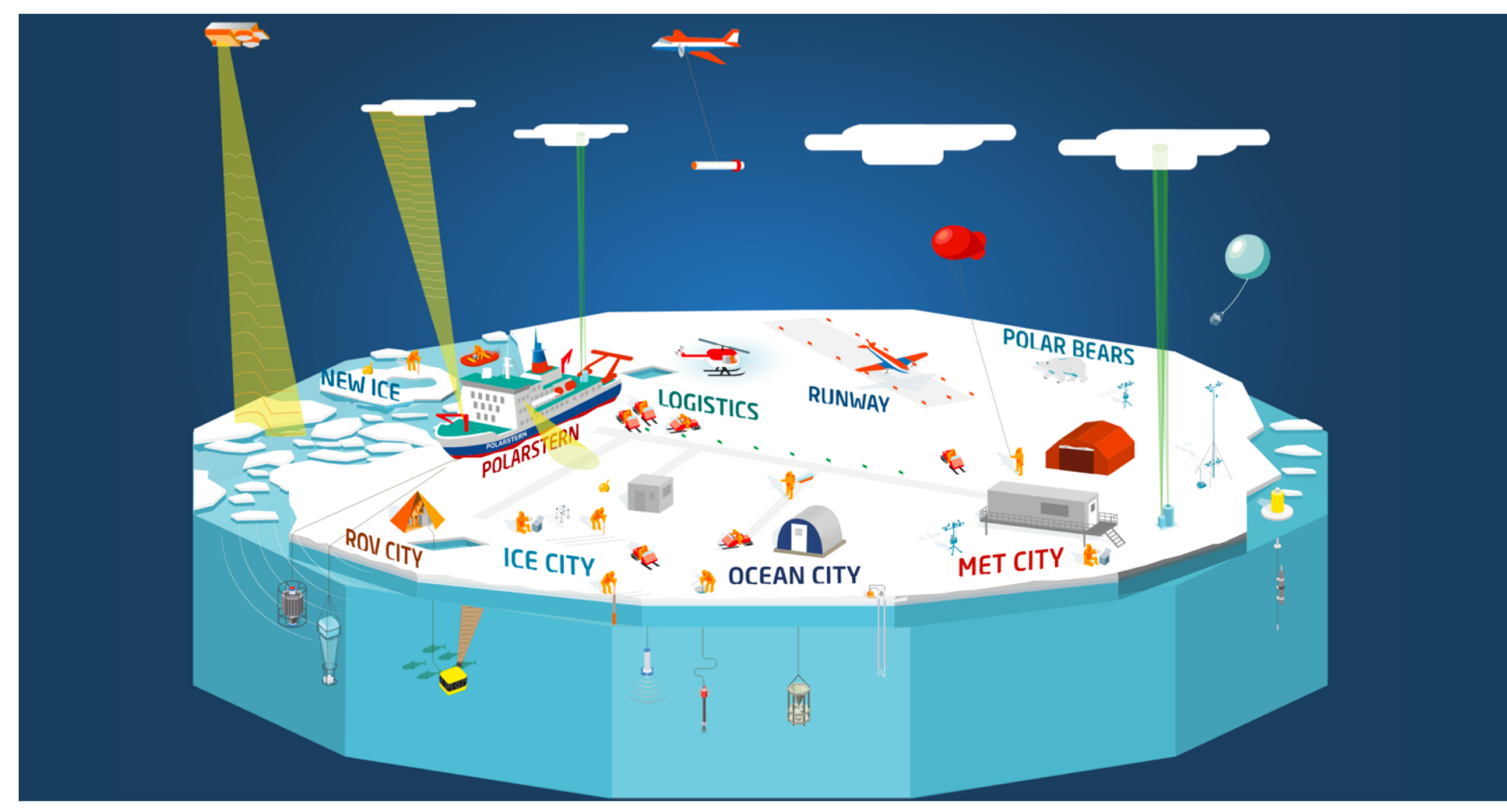


MOSAiC ernational Expedition $\square$

Main scientific focus areas

ब्ANI

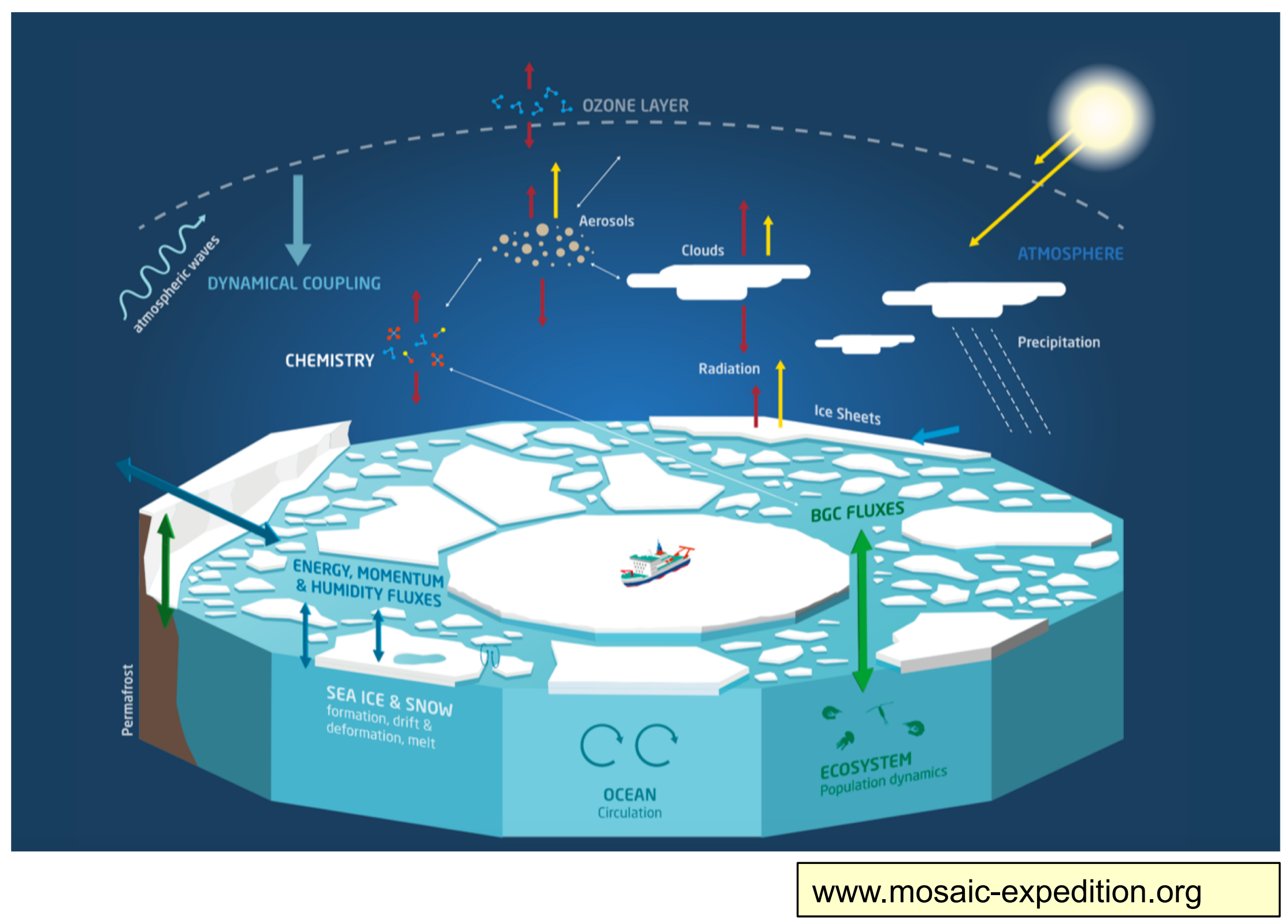

HELMHOLTZ 
MOSAiC International Arctic Drift

Expedition

\section{MOSAiC Data Policy}

\section{Expedition Start 19.09.2019}

- First data back on land: January 2020

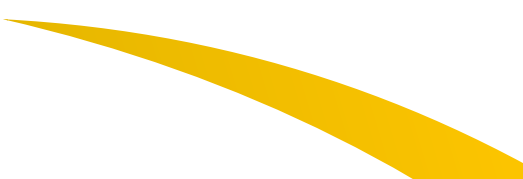

January 2021

- All Sensor Data on MCS

- fast analysis sample data on MCS

- primary subset of laboratory data on MCS

\section{Expedition End: October 2020}

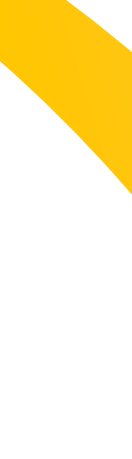

- project's success relies upon professional coordination and data sharing across the participants

- transparent Data Policy is essential to achieve MOSAiC science objectives, to facilitate collaboration, and to enable broad use and impact of the MOSAiC data legacy

data on MCS

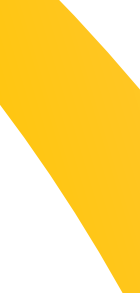

January 2022

- full collection of laboratory data on MCS

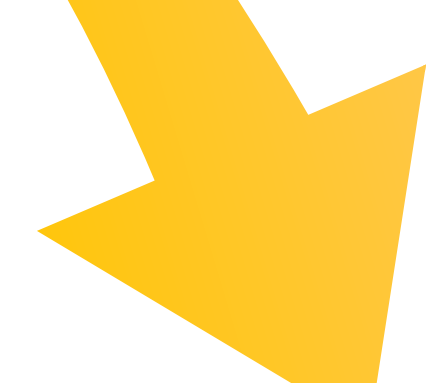

Public release: 01.01.2023 or earlier!

\section{MOSAiC Central} Storage (MCS)

- Aboard Polarstern: basis for gathering data along the year of operation

- On land: central and reliable storage and working database of MOSAiC data. 


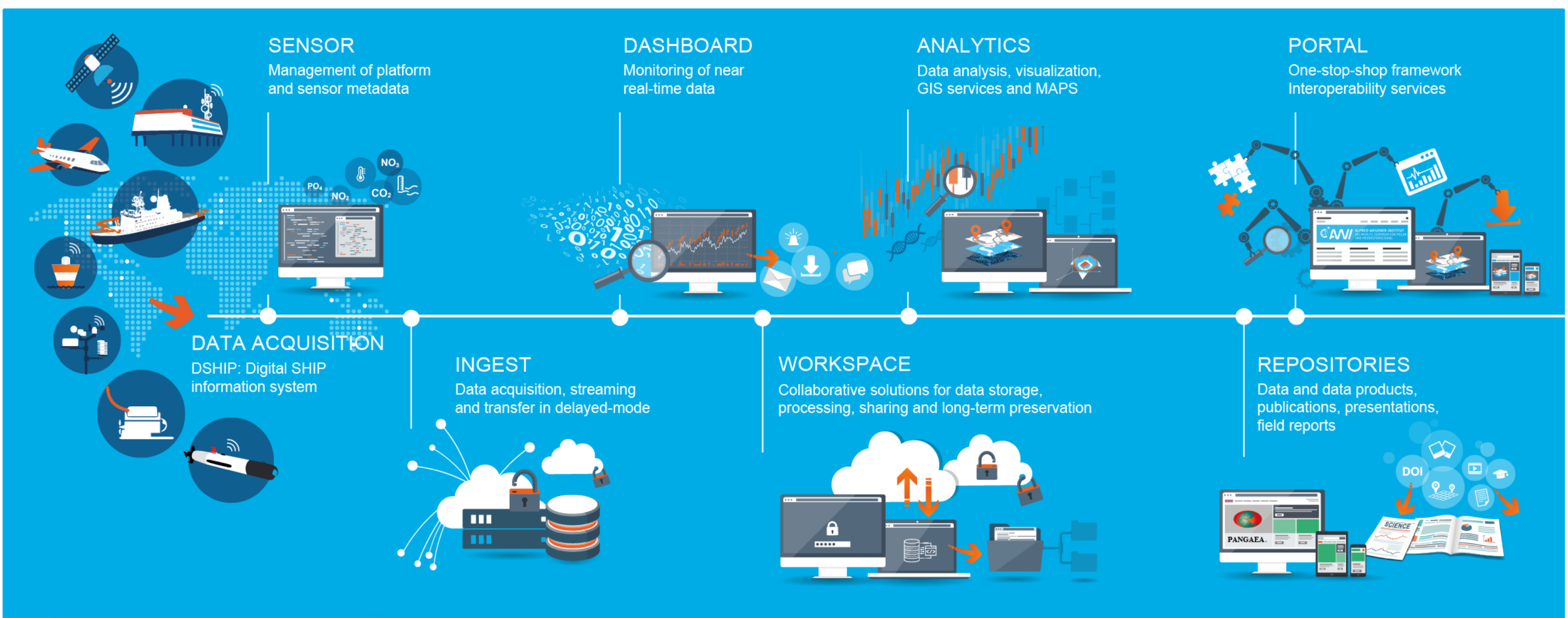

Metadata

Description 
MOSAiC ternational Arctic Drift

Expedition T1
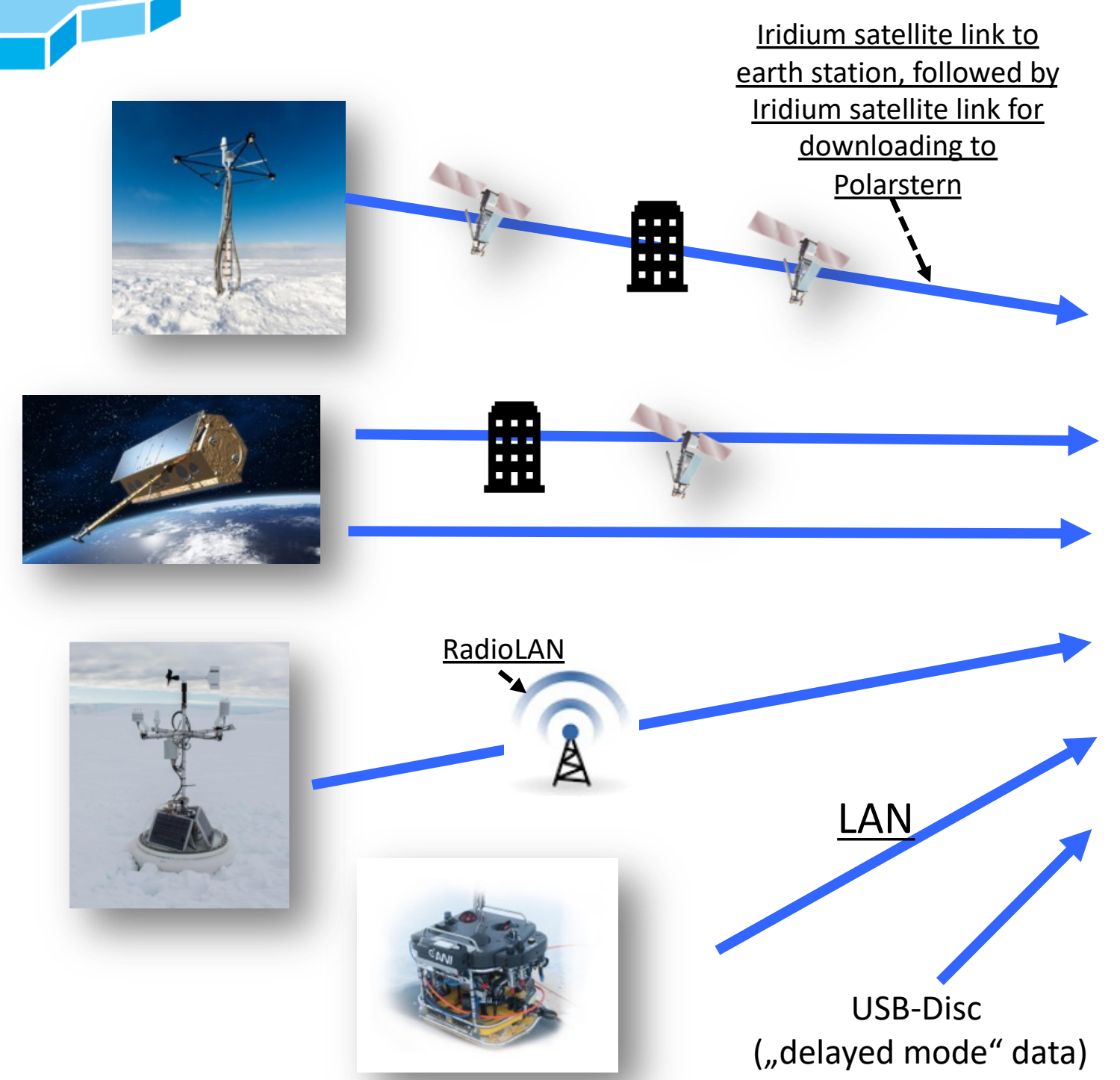

(,"delayed mode“ data)

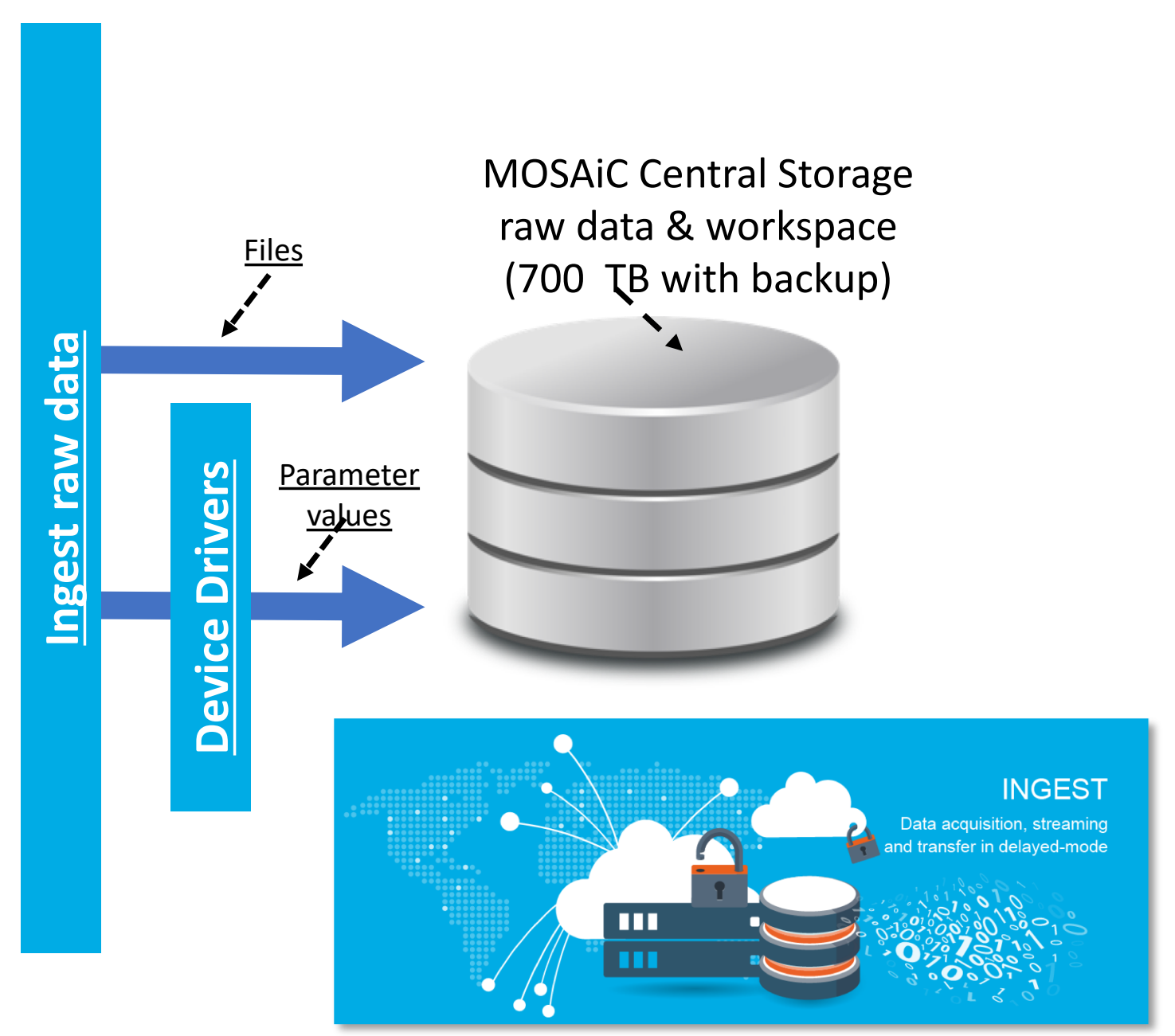



SENSOR.awi.de
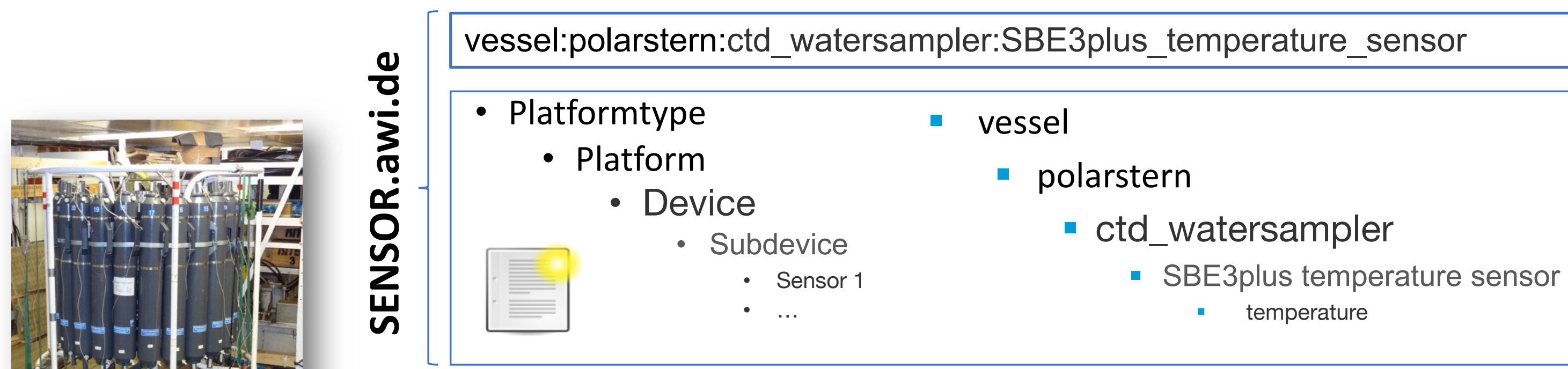

- vessel/polarstern/ctd_watersampler/SBE3plus_temperature_sensor/ DEVICEOPERATION_ID.../SensorFile.xxx

- vessel/polarstern/ctd_watersampler/SBE3plus_temperature_sensor/ DATE.../File.xxx

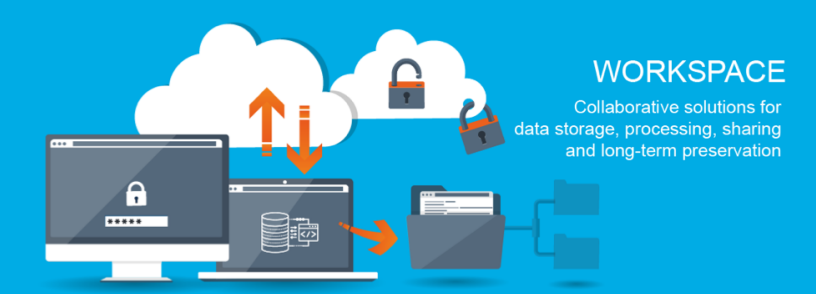

vessel:polarstern:ctd_watersampler:SBE3plus_temperature_sensor: temperature 

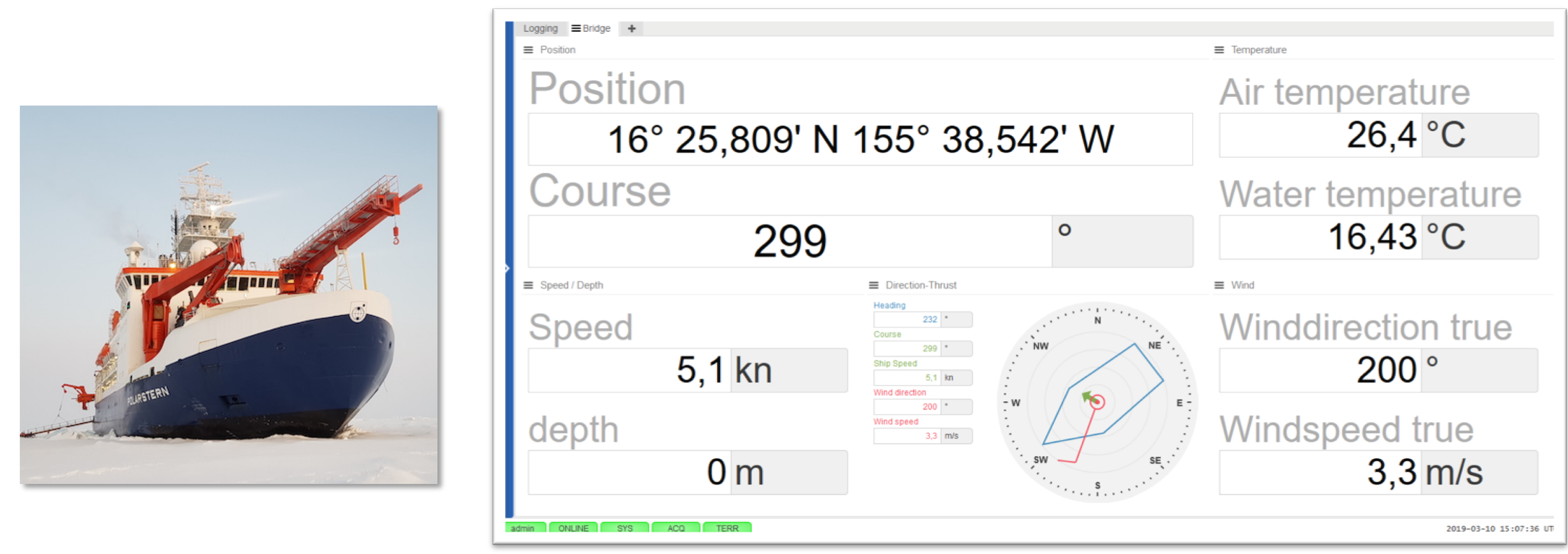

- werum.de 


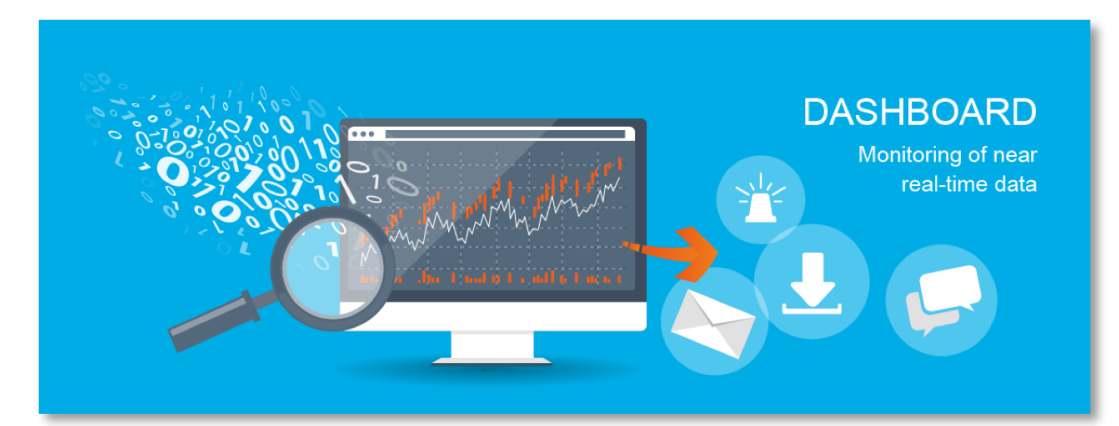

\section{(OINI}

RV Polarstern

$-64.5552-56.4124$

\section{$\mathbb{U} \mathbf{A V} /$ Dashboar
Test for MOSAic Drift Station}

MOSAiC

$\underset{\substack{\text { Arctic Oritt } \\ \text { Expedition }}}{\text { Interation }}$

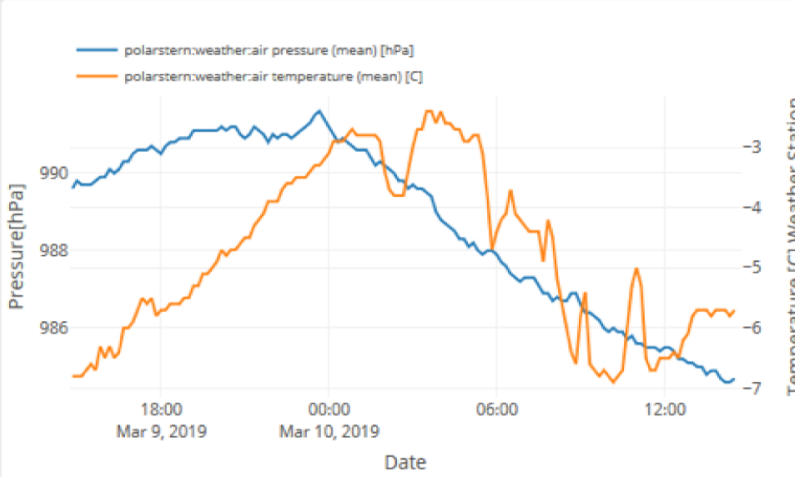

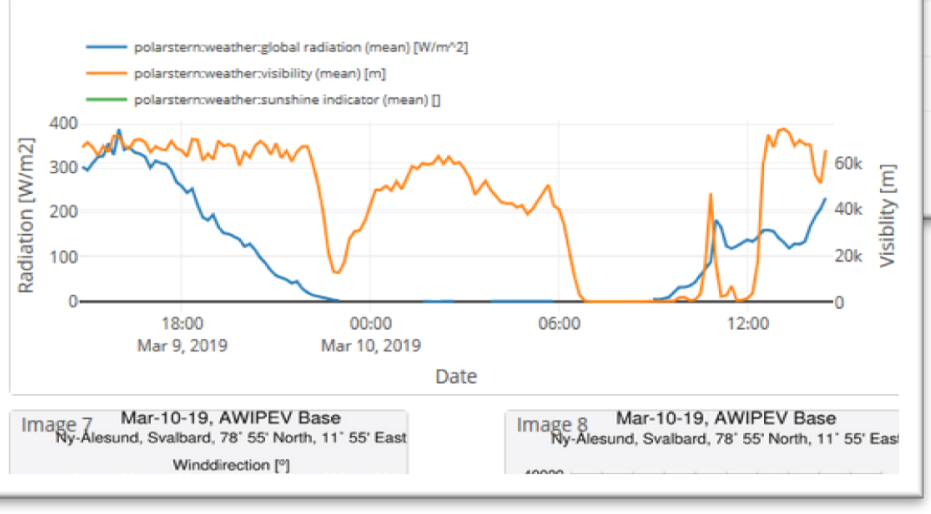

Data
Data

Monitoring 
MOSAiC

Expeditio
- ...browser based mapping and viewing system

- decision support on board

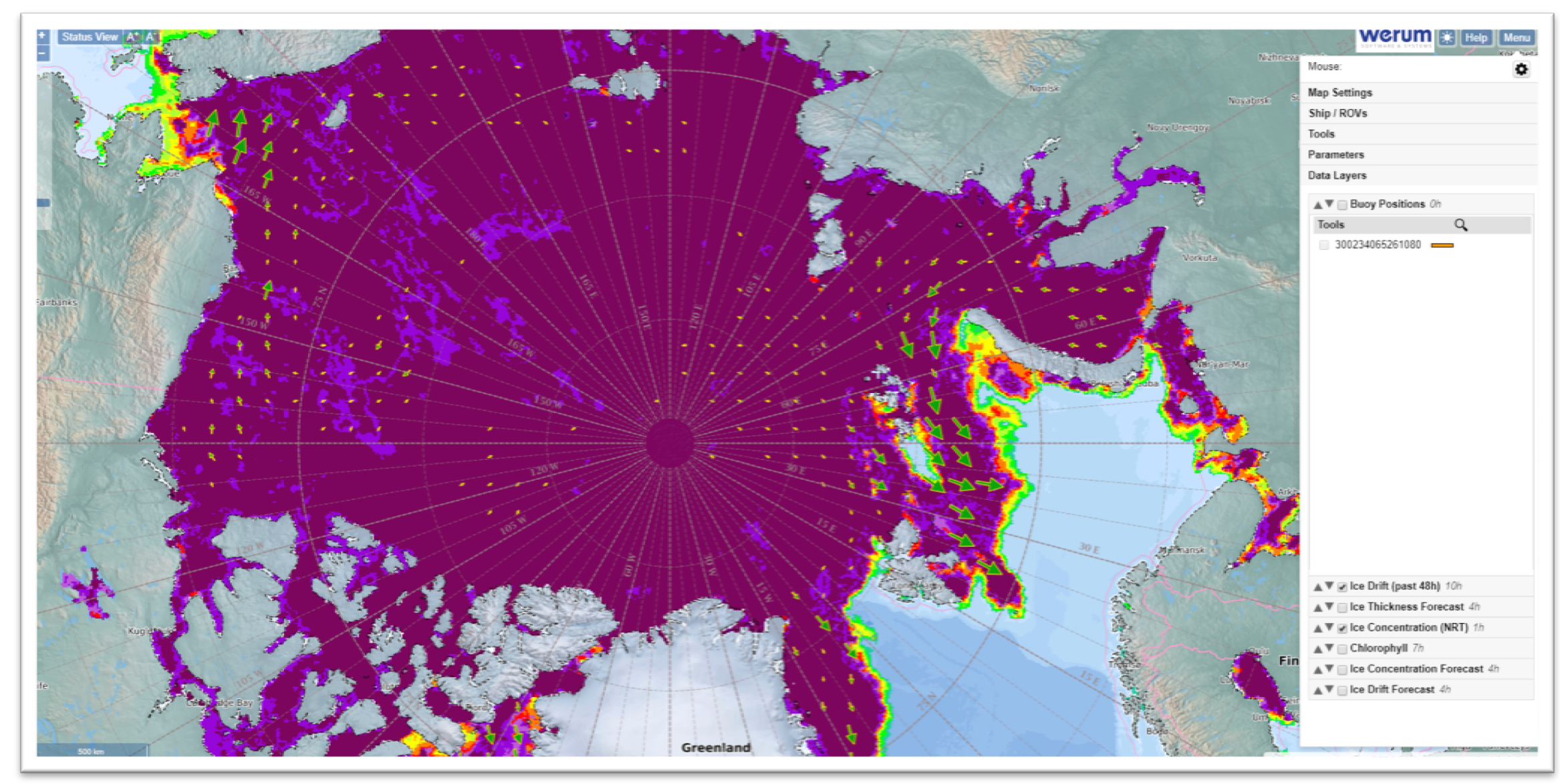

werum

werum.de 

machine different operating systems and development environments

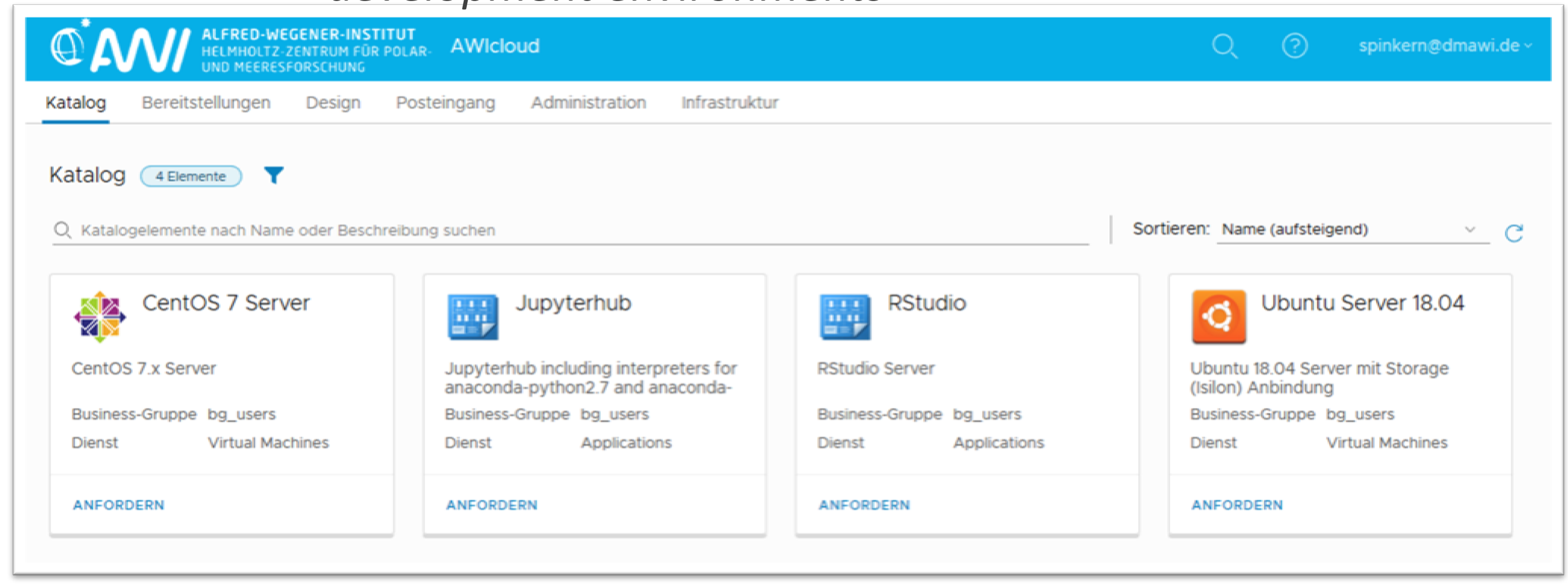

mware 
MOSAiC

Arctic Drift

Expedition

- pre-installed environments

- access to persistent storage

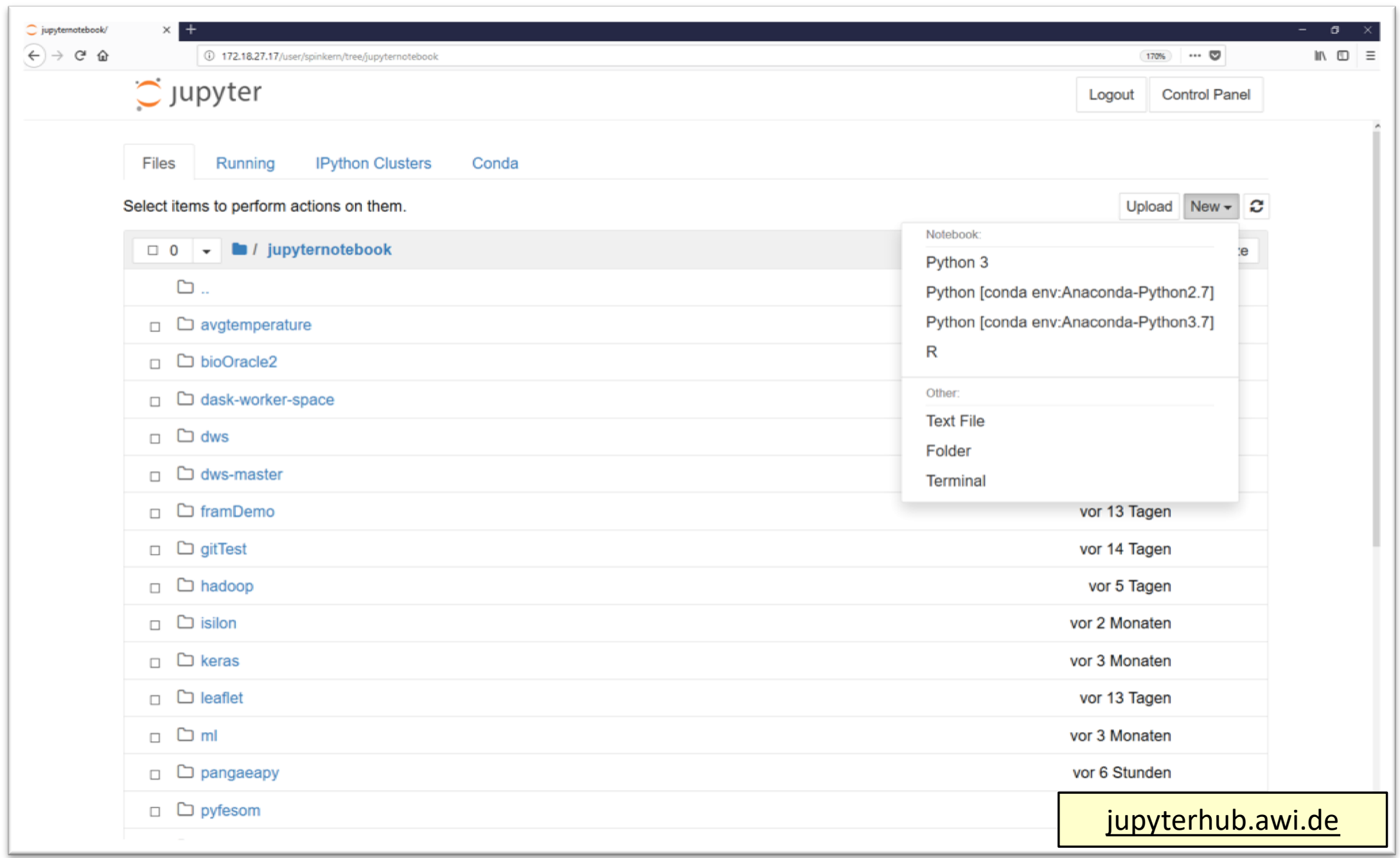

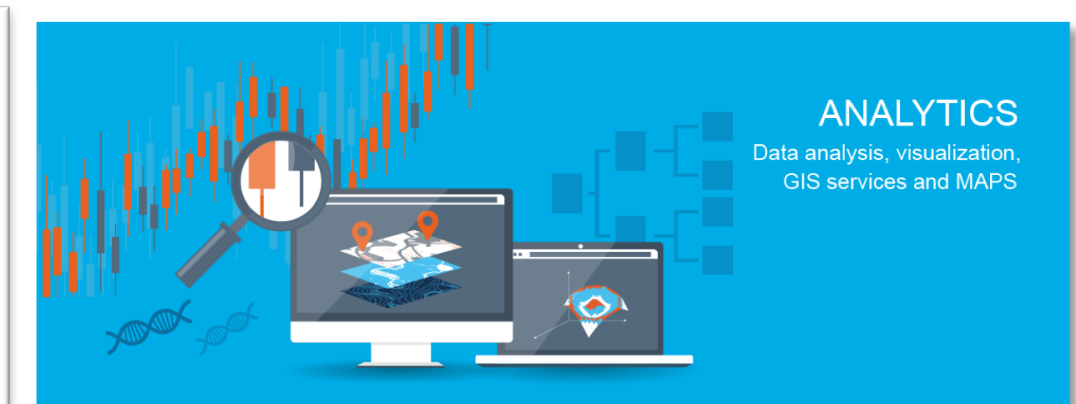




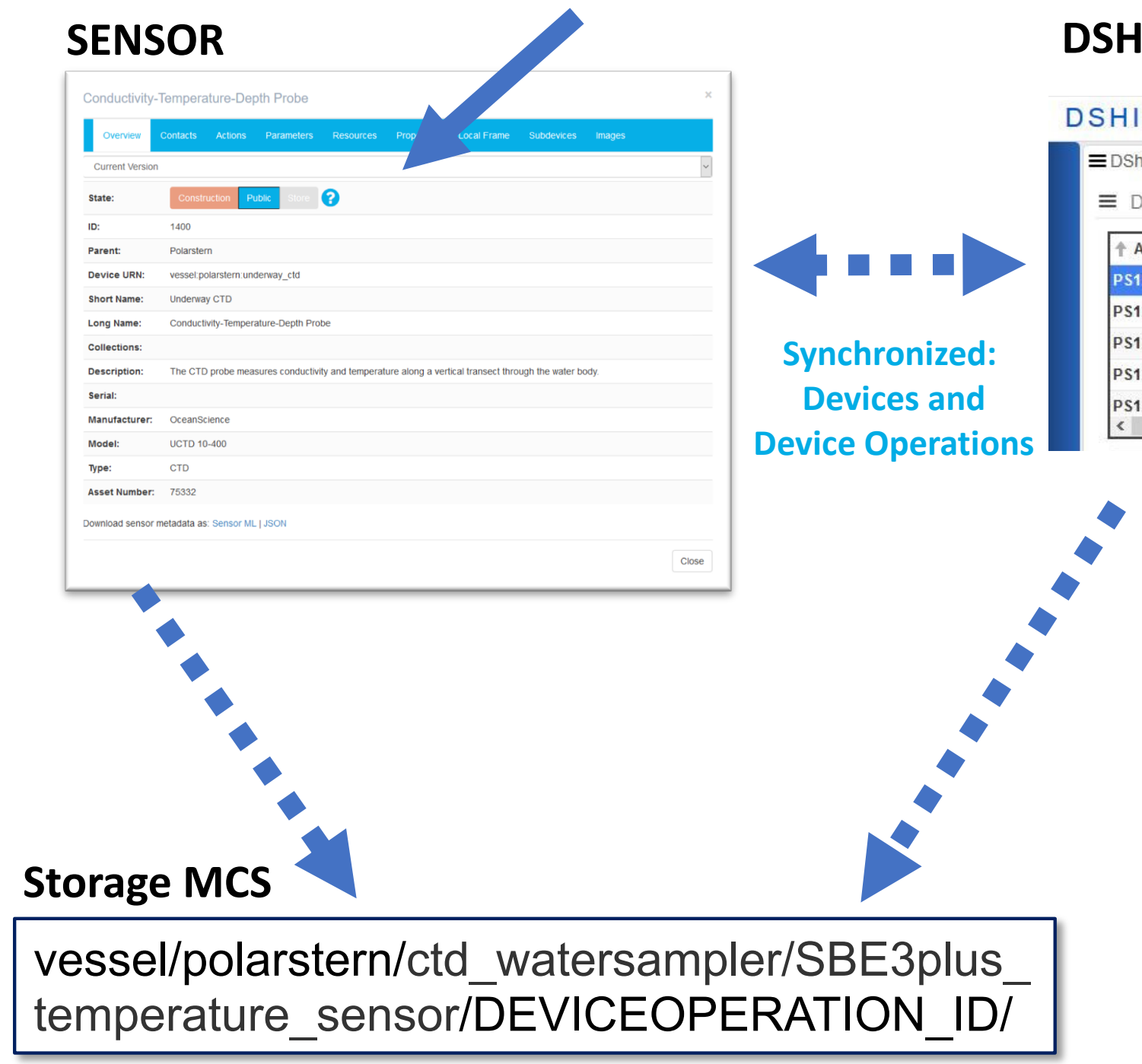

\section{DSHIP-ActionLog}

SHIP MOSAiC20192020/1 || $75^{\circ} 08,241^{\prime}$ N 039 07,762' E || 2019-09-22 ... werum EDShip_ActionLog

\begin{tabular}{|c|c|c|c|c|c|c|c|c|}
\hline$\uparrow$ Activity - Device Operation $\bar{\nabla}$ & Timestamp & Device & Action & Latitude & Longitude & Depth (m) & Speed $(\mathrm{kn})$ & \\
\hline PS12221__4-1 CTD_SBE9plus_485 & 22.09.2019 09: & |Conductivity-Te. & |information & $75^{\circ} 07,932^{\prime} \mathrm{N}$ & $039^{\circ} 05,969^{\prime} \mathrm{E}$ & | 184.0 & | 13.1 & \\
\hline PS122/1_3-1 topAWI & 22.09.2019 09: & Towed Ocean .. & information & $75^{\circ} 05,362^{\prime} \mathrm{N}$ & $038^{\circ} 51,058^{\prime} \mathrm{E}$ & 186.0 & 13.3 & \\
\hline PS122/1_2-1 AIRGUN_PS & 22.09.2019 08: & Air Gun & profile start & $74^{\circ} 59,125^{\circ} \mathrm{N}$ & $038^{\circ} 15,496^{\prime} \mathrm{E}$ & 177.0 & 13.3 & \\
\hline PS12211_1-1 topAWI & 21.09.2019 18: & Towed Ocean . & information & $73^{\circ} 08,358^{\prime} \mathrm{N}$ & $029^{\circ} 49,934^{\prime} \mathrm{E}$ & 267.0 & 12.7 & \\
\hline PS12211_0_Underway-60 Weather & 20.09.2019 17: & Weather Station & station start & $69^{\circ} 40,773^{\prime} \mathrm{N}$ & $018^{\circ} 59,799^{\prime} \mathrm{E}$ & 0.0 & 0.0 & \\
\hline
\end{tabular}

Log device operations: Directly in DHSIP,

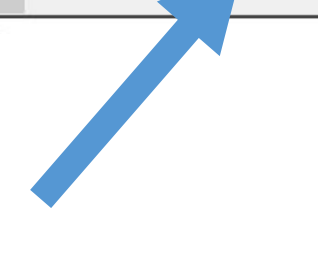

or via import from log files

(e.g generated by IceFloeNavi-App)

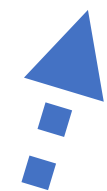
temperature_sensor/DEVICEOPERATION_ID/ 
MOSAiC ernational

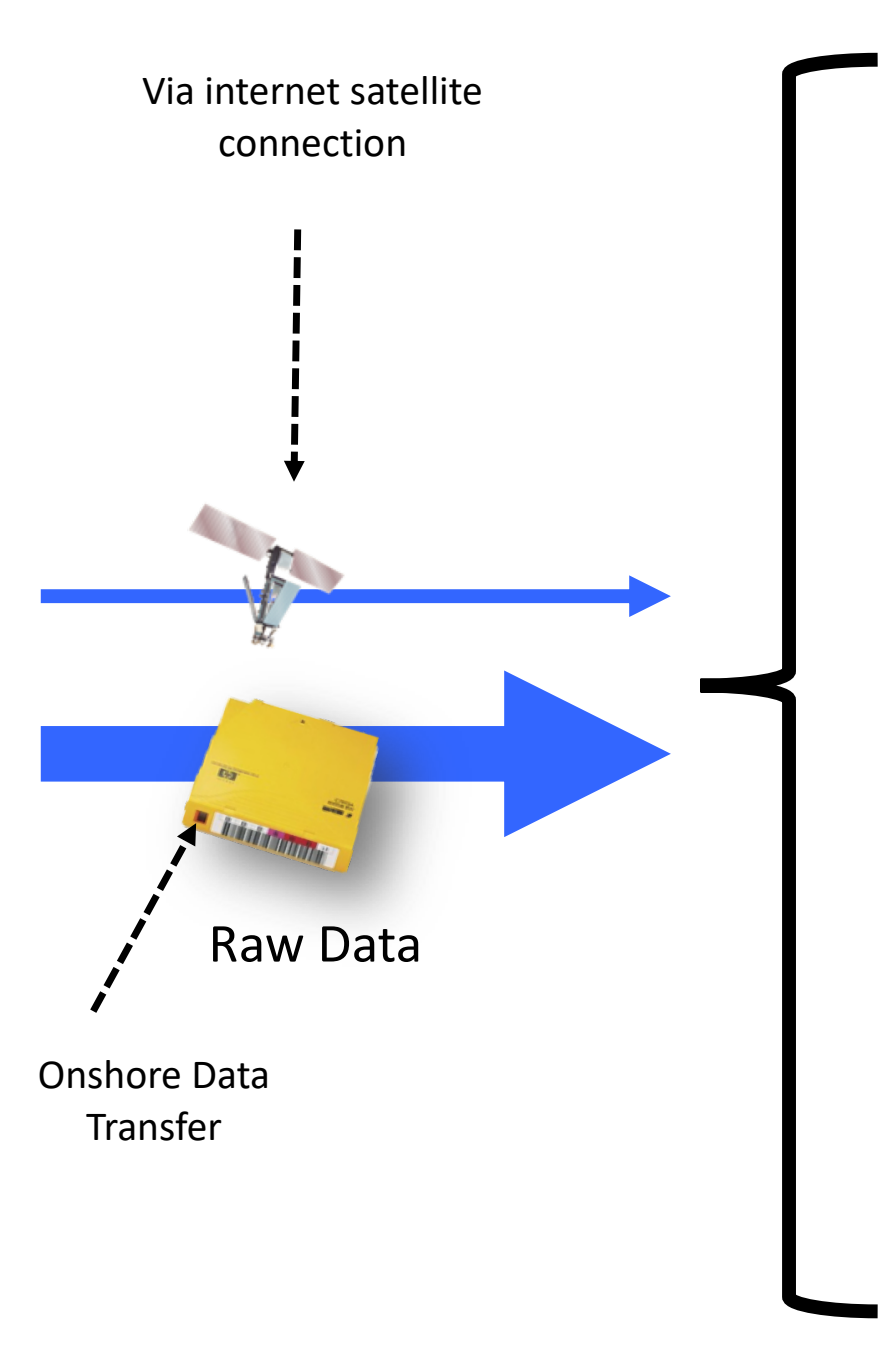

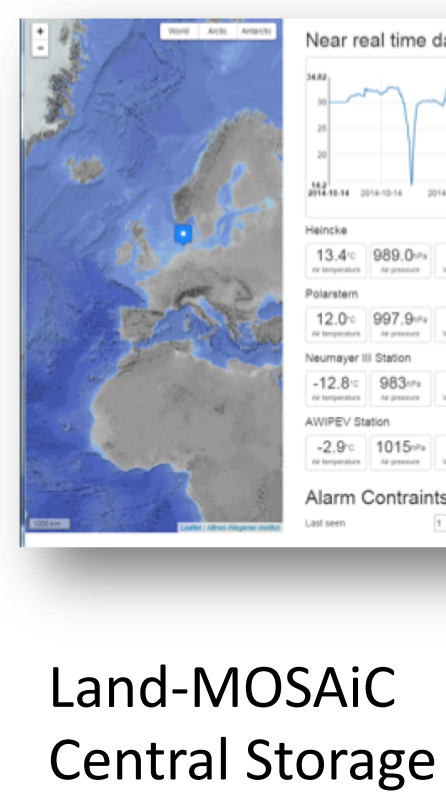
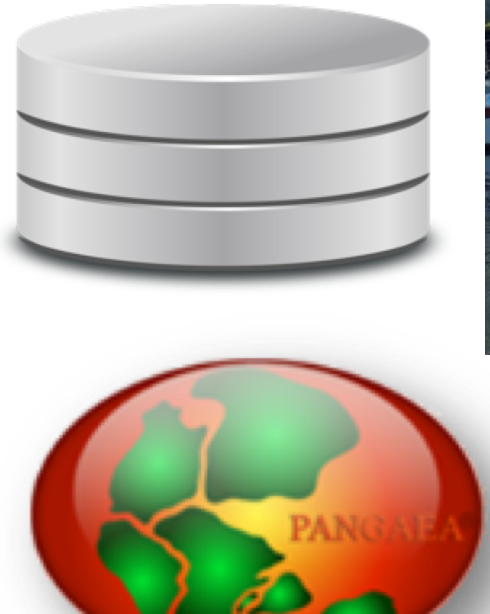

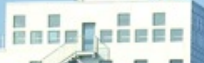

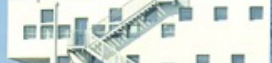

- $50=1$
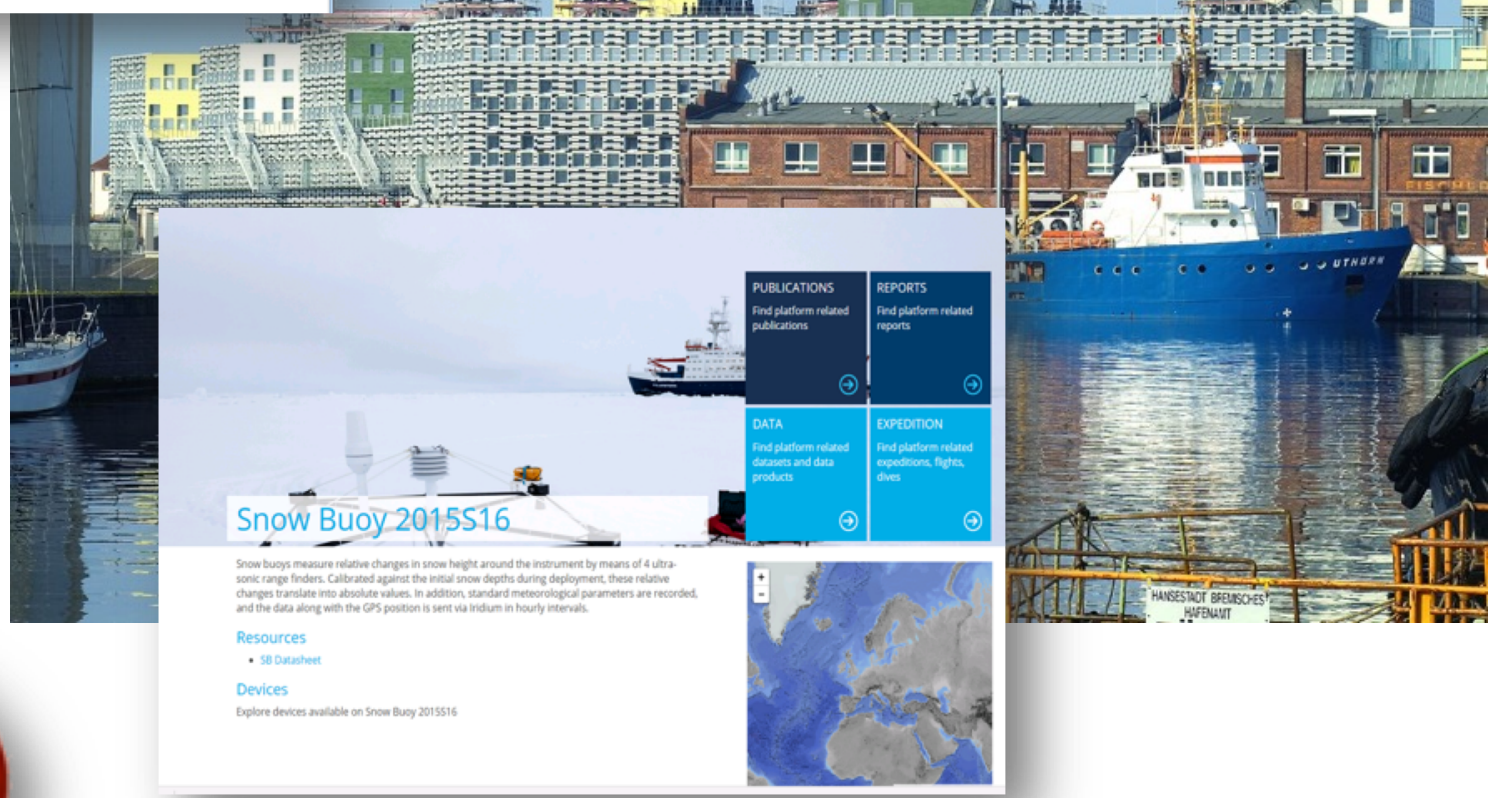

HELMHOLTZ 


\section{PANGAEA.}

Data Publisher for Earth \& Environmental Science

Rex, Markus (2020): Links to master tracks in different resolutions of POLARSTERN cruise PS122/1, Troms $~-$ Arctic Ocean, 2019-09-20 - 2019-12-13. Alfred Wegener Institute, Helmholtz Centre for Polar and Marine Research, Bremerhaven,

Aways quote above citation when using datal You can downlload the citation in several formats below

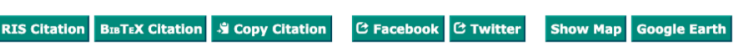

Median Latitude: 84.972877 * Median Longitude: 120.353461 * South-bound Latitude: 69.679426 * West-bound Longitude: 18.996638 * North-bound Latitu DaterTime Start: 2019-09-20117:50:00 * DaterTime End: 2019-12-13T09:04:45

PS12211-track Q * Latitude Start: 69.679550 * Longitude Start: 18.996650 * Latitude End: $86.593190 *$ *ongitude End: 119.241500 * DaterTime Start: 201 Campaign: PS12211 (MOSAiC20192020) $Q$ * Basis: Polarstern Q * Method/Device: Underway cruise track measurements (CT) Q * Comment: Troms
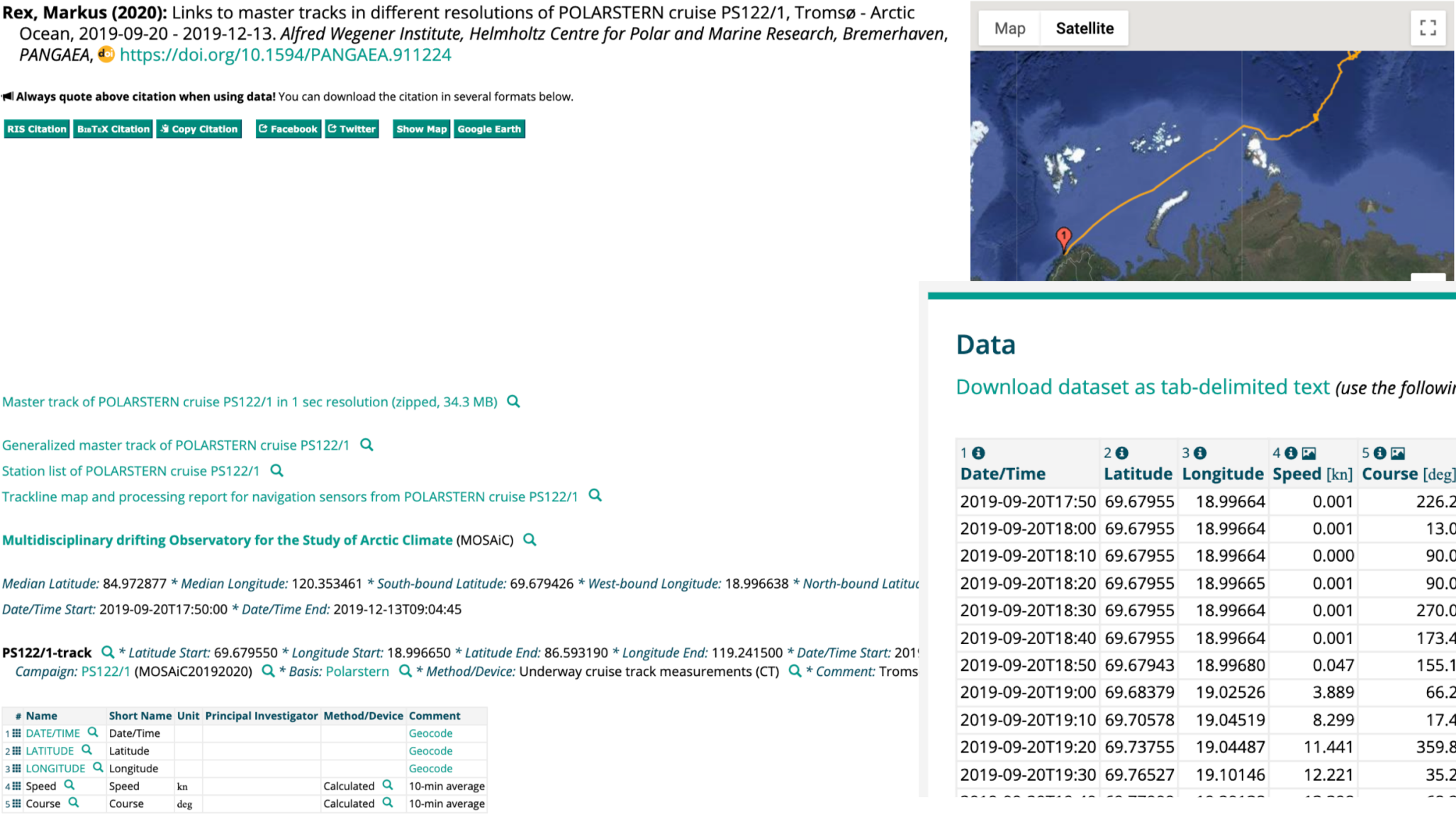

Data

Download dataset as tab-delimited text (use the following character encoding: UTF-8: Unicode (PANGAEA default)

$\begin{array}{llll}10 & 20 & 30 & 40\end{array}$ 2019-09-20T17:50 69.67955 $18.99664 \quad 0.001$ 2019-09-20T18:00 $69.67955 \quad 18.99664 \quad 0.001$ 2019-09-20T18:10 $69.67955 \quad 18.99664$ 2019-09-20T18:20 $69.67955 \quad 18.99665$ 2019-09-20T18:30 69.67955 18.99664 2019-09-20T18:40 69.67955 18.99664 2019-09-20T18:50 $69.67943 \quad 18.99680$ 2019-09-20T19:00 $69.68379 \quad 19.02526$ 2019-09-20T19:10 69.70578 19.04519 2019-09-20T19:20 $69.73755 \quad 19.04487$ 2019-09-20T19:30 69.76527 19.10146

\begin{tabular}{|r|r|}
\hline 0.001 & 226.2 \\
\hline 0.001 & 13.0 \\
\hline 0.000 & 90.0 \\
\hline 0.001 & 90.0 \\
\hline 0.001 & 270.0 \\
\hline 0.001 & 173.4 \\
\hline 0.047 & 155.1 \\
\hline 3.889 & 66.2 \\
\hline 8.299 & 17.4 \\
\hline 11.441 & 359.8 \\
\hline 12.221 & 35.2 \\
\hline
\end{tabular}




\section{MOSAiC}

nternational

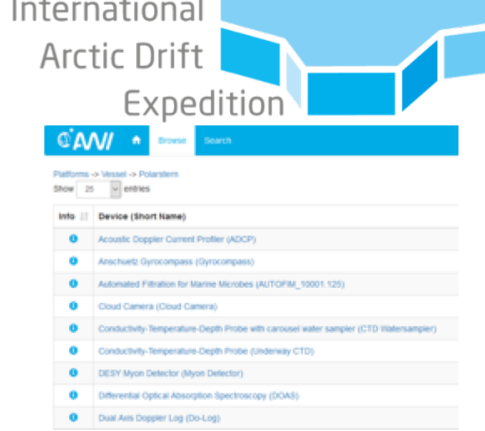

SENSORWeb at AWI and onboard of Polarstern

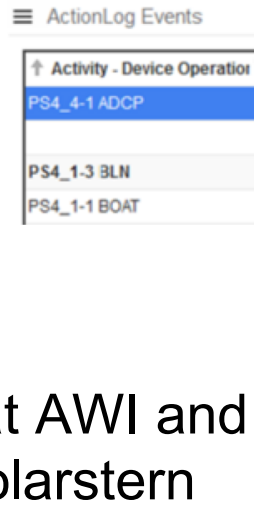

Acquisition organized by MOSAiC groups.

DSHIP-ActionLog for

Device-Operation ID

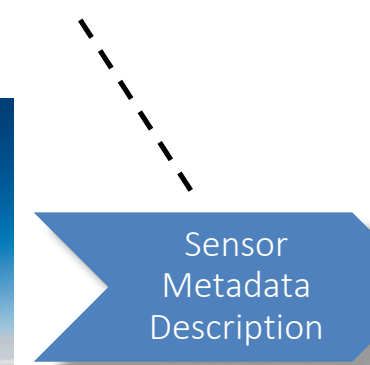
management

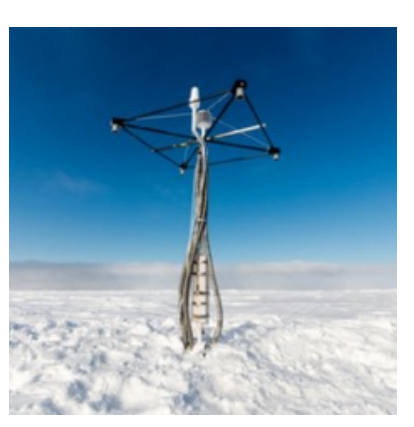

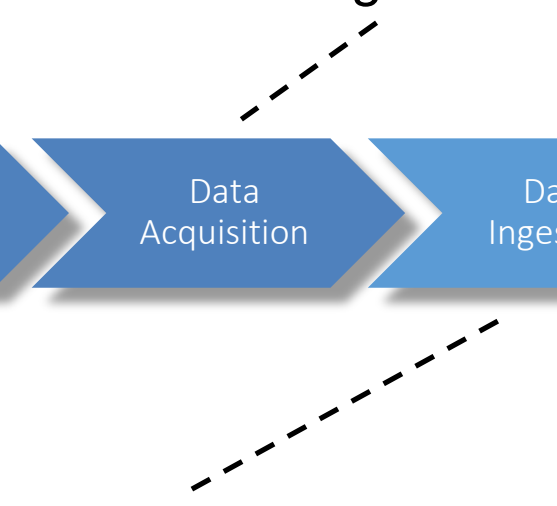

Data transfer via satellite, local LAN, radio LAN as stream and/or in delayed mode
MOSAiC Central Storage and workspace
$0.81,0.00$

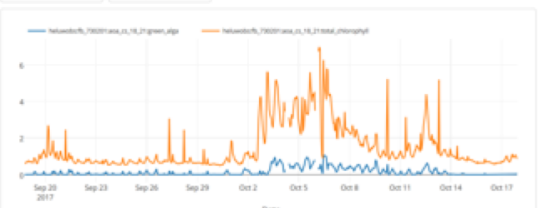

\section{CANI}

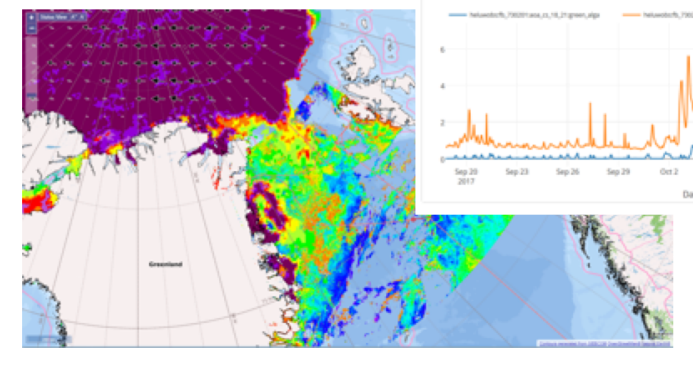

DSHIP-Mapviewer and DASHBOARD at AWI and onboard of Polarstern
Raw and primary data archiving at AWI. Data transfers after legs or parts during legs

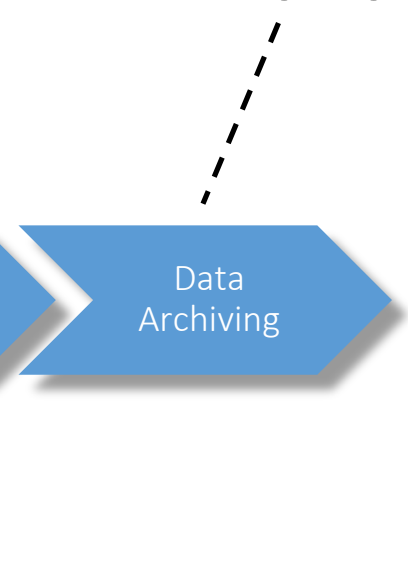

Using workspace and Marketplace (VM) e.g. with Jupyter Notebook (R or Python) or Bash-Script or or ...?

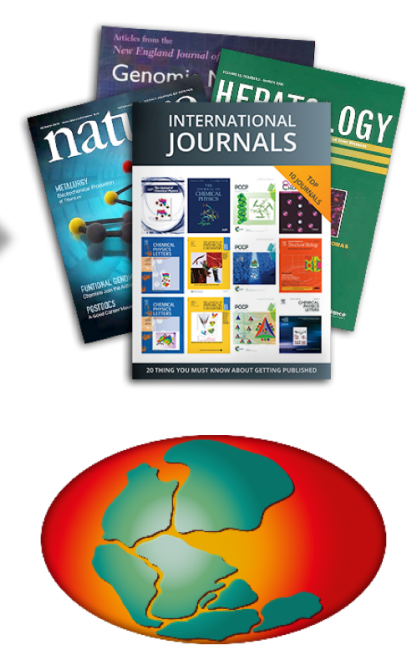

